Gilbert Lelord Jean-Pierre Muh Dominique Sauvage Josiane Hérault

\section{ADRESSE}

G. Lelord: professeur émérite, physiologie. J.P. Muh: professeur. J. Hérault: maître de conférences, biochimie et biologie moléculaire.

D. Sauvage: professeur, pédopsychiatrie. Inserm U.316, CHU Bretonneau, 2, boulevard Tonnellé, 37044 Tours Cedex, France.

\title{
Neurobiologie des syndromes autistiques de l'enfant
}

On a longtemps considéré que l'autisme de l'enfant, caractérisé par un isolement, des difficultés de communication et des réactions bizarres à l'environnement, pouvait être dû à des erreurs conscientes ou inconscientes de la mère. En fait, des enquêtes épidémiologiques, des recherches neurobiologiques portant notamment sur l'association de syndromes autistiques à des maladies connues (génétiques et/ou métaboliques), et des études cliniques sur les signes très précoces suggèrent que l'autisme serait lié à un trouble du développement du système nerveux. Les moyens actuels d'exploration (imagerie cérébrale, étude du métabolisme des neurotransmetteurs, études génétiques) ont permis de mettre en évidence des anomalies très diverses. Ces résultats préliminaires n'ont pour le moment pas de conséquence thérapeutique ; en revanche, les données portant sur la physiologie cérébrale permettent d'orienter les «thérapeutiques d'échange et de développement", psychothérapies éducatives qui contribuent à l'amélioration de ces enfants.

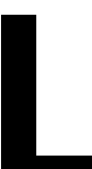

e vocable autisme, utilisé pour la première fois en 1911 par Bleuler [1], psychiatre d'adultes, désignait un ensemble de signes caractérisés par le repli sur soi, l'isolement et les réactions de retrait vis-à-vis de l'entourage. Mais c'est Kanner [2] qui, le premier, a décrit en 1943 le tableau clinique de l'autisme de l'enfant. Cette affection touche environ 4 enfants pour 10000 dans les formes dites «pures »; 20 pour 10000 dans les formes associées à d'autres handicaps. La description de Kanner reste valable aujourd'hui. L'enfant présente une «incapacité d'établir des relations", il se replie «comme dans une coquille », il éprouve la plus grande difficulté à s'exprimer par la voix, les gestes, la mimique; souvent même, il ne parle pas, il a horreur des bruits forts; ses gestes sont souvent bizarres, répétés de façon inlassable; enfin, il a besoin d'un environnement immuable et se montre très perturbé par le moindre changement. Ces signes apparaissent chez le jeune enfant qui ne répond pas aux sollicitations de sa mère. Il est très émouvant, et pour les parents très éprouvant, de se trouver en présence d'un enfant qui évite tout contact, qui ne cherche pas à communiquer, 
qui ne sourit pas, ne gazouille pas, ne tend pas les bras et qui, très facilement contrarié, ne s'exprime que par des cris. Kanner attribuait cet autisme de l'enfant à «une incapacité innée d'établir le contact affectif habituel avec les personnes». Une telle conception était alors déroutante. En effet, les connaissances sur le système nerveux portaient essentiellement sur les systèmes de la sensibilité, de la motricité, des organes des sens, ou à la rigueur des centres du langage. Or ces enfants autistes ne sont pas paralysés, ne sont pas sourds et, pour certains d'entre eux, sont capables d'articuler certains mots ou phrases «en écho». Parallèlement, la plupart des observations effectuées au même moment chez l'enfant montraient que la privation précoce de la présence maternelle entraînait des troubles sévères du comportement. Aussi Kanner lui-même parlaitil quelques années plus tard de la "froideur affective" des mères et, pendant de longues années, cette conception de l'origine maternelle de l'autisme, constamment défendue par Bettelheim [3], a prévalu.

3. Bettelheim B. La forteresse vide: l'autisme infantile et la naissance du soi. Paris: Gallimard, 1973.

4. Lelord G, Hérault J, Perrot A, et al. L'autisme de l'enfant, une déficience relationnelle liée à un trouble du développement du système nerveux central. Bull Acad Natl Med 1993; 177: 1423-32.

5. Lelord G, Sauvage D. L'autisme de l'enfant. Paris: Masson, 1991.

6. Smalley SL, Asarnow RF, Spence MA. Autism and genetics. A decade of research. Arch Gen Psychiatr 1988; 45: 953-61.

7. Fombonne E, du Mazaubrun C. Prevalence of infantile autism in four French regions. Soc Psychiatr Epidemiol 1992; 7, 27 : 203-10.

8. Ritvo ER, Freeman BJ, Pingree C, et al. The UCLA University of Utah epidemiologic survey of autism: prevalence. Am J Psychiatr 1989; 146: 194-245.

9. Folstein SE, Piven J. Etiology of autism. Genetic influences. Pediatrics 1991; 87: 767-73.

10. Henry I. Neurofibromatose de type 1 ou maladie de von Recklinghausen: de nombreuses questions restent posées. médecine/sciences 1995; 11 : 93-8.

11. Gillberg C, Coleman M. The biology of autistic syndromes, $2^{\text {nd }}$ ed. London: Mac Keith, 1992.

12. Smalley S, Tanguay P, Smith M, Gutierrez G. Autism and tuberous sclerosis. $J$ d'épilepsie peuvent être présents avec un pourcentage variant suivant les auteurs de 5 à $30 \%$ [5].

Des arguments non négligeables dans le déterminisme de l'autisme font envisager le rôle de facteurs génétiques $[5,6]$. Certains de ces facteurs sont déjà bien connus : la prévalence identique de l'autisme dans différentes populations [7], le sex-ratio (en faveur des garçons), les cas familiaux (surtout si l'on considère aussi d'autres formes associées de troubles du développement) [8], les résultats très significatifs des études de jumeaux: si l'un des jumeaux est autiste, le risque pour le jumeau du même œuf dépasse $80 \%$ [9].

Outre ces données épidémiologiques, le second type d'arguments en faveur de facteurs génétiques est celui de cas relativement fréquents de syndromes d'autisme associés à une maladie dont le déterminisme génétique est connu. Deux affections neurologiques à transmission génétique et appartenant au groupe des syndromes neurocutanés, la neurofibromatose de von Recklinghausen et la sclérose tubéreuse de Bourneville sont retrouvés associés à l'autisme. La neurofibromatose de type I (NF1) ou neurofibromatose de von Recklinghausen a une prévalence de 1 pour 2000 à 3000 individus. Son gène a été localisé en 17q11.2 [10]. La transmission de la maladie s'effectuerait selon le mode dominant. Gillberg et Coleman [11] signalent que $6 \%$ d'enfants autistes présentent cette maladie. La sclérose tubéreuse de Bourneville, bien que moins fréquente que la neurofibromatose $(0,5$ à 1 pour 10000 naissances) donne souvent des troubles du développement plus évidents $\left(\mathrm{m} / \mathrm{s} n^{\circ} 5\right.$, vol. $10, p .601$; $n^{\circ} 4$, vol. 11, p. 436). Des études de liaison ont montré une hétérogénéité génétique. En effet, différentes régions chromosomiques sembleraient être impliquées dans l'apparition de la maladie. La présence de la sclérose tubéreuse de Bourneville chez les autistes varie de 0,4 à $3 \%$ [12] mais il semblerait que cette association soit plus importante chez les filles autistes. Parmi les maladies métaboliques, c'est la phénylcétonurie qui est la plus connue pour être retrouvée dans des observations d'autisme. Cette maladie, à transmission autosomique récessive, est due à 
une déficience en phénylalanine-4hydroxylase, enzyme qui convertit la phénylalanine en tyrosine [13]. Cette aminoacidopathie se caractérise par un retard mental, des troubles neurologiques et des troubles du comportement. Le gène de cette enzyme a été localisé sur le chromosome 12. Quelques études ont décrit des populations d'autistes avec phénylcétonurie et Chen et Hsiao [14] ont rapporté un cas de phénylcétonurie à symptomatologie autistique presque exclusive. L'association de l'autisme a été également retrouvée avec certaines anomalies chromosomiques comme, par exemple, la présence de chromosomes surnuméraires au niveau des chromosomes sexuels ainsi que des anomalies autosomiques telles que la trisomie 21. Par ailleurs, l'intérêt des dernières années s'est porté sur la fragilité du chromosome X [15]. Les évaluations de la proportion d'enfants ayant un $\mathrm{X}$ fragile parmi les enfants autistes donnent des résultats qui varient de 0 à $16 \%$. Un biais de recrutement peut expliquer ces chiffres discordants qui apparaissent dans certaines publications qui incluent les comportements autisticlike des garçons $\mathrm{X}$ fragile. La lésion spécifique du syndrome $\mathrm{X}$ fragile (ou syndrome de Martin-Bell) est localisée en Xq27.3 ( $\mathrm{m} / \mathrm{s}^{\circ} 6$, vol. $\left.7, p .637\right)$ [16] et correspond à une modification du gène FMR hyperméthylé [17] avec présence de triplets CGG répétés plus de 50 fois pour la prémutation et plus de 200 fois pour la mutation complète [18].

D'autres associations sont possibles avec l'autisme, la plus marquante étant le syndrome de Rett. Ce syndrome, avant son individualisation clinique, était inclus dans les syndromes autistiques. Il atteint exclusivement les filles (1 à 2 sur 15000) avec une concordance absolue chez les jumelles monozygotes, mais pas de concordance chez les dizygotes. Deux translocations de la partie autosomique de l'X ont été décrites $(\mathrm{t}(\mathrm{X} ; 3)$ et $\mathrm{T}(\mathrm{X} ; 22))$. La cause de ce syndrome reste à ce jour inconnue mais l'hypothèse généralement proposée est qu'il s'agirait d'une maladie liée à l'X entraînant la létalité chez les garçons [19]. Il existe également une association importante entre l'autisme et le retard mental. En effet, le pourcentage d'enfants retardés mentaux dans des populations d'autistes varie de 65 à $85 \%$. L'exploration des syndromes autistiques ne fait que commencer. Il faut effectuer dès à présent la recherche de la présence d'un $\mathrm{X}$ fragile et faire un examen cytogénétique en plus des examens cliniques et métaboliques approfondis afin de pouvoir différencier un retard mental avec ou sans autisme.

\section{Atteintes des fonctions neuropsychologiques}

L'examen médical comporte nécessairement le colloque singulier avec l'enfant et sa famille. Mais il s'efforce en outre de déceler et d'évaluer des déficiences [5]. L'étude fine des troubles de la communication à l'aide du magnétoscope et le recours aux échelles d'évaluation [20] ont permis de préciser à côté des signes habituellement décrits dans l'autisme (retrait, troubles des relations avec autrui) des troubles de l'adaptation de l'enfant aux stimulations de l'environnement: anomalies de l'attention sélective, particularités du regard, bizarreries de l'audition (impression de surdité contrastant avec une sensibilité excessive à certains sons, difficultés à recevoir et à lancer le ballon...) (Tableau I). L'analyse statistique d'un nombre suffisant d'observations quantifiées permet non seulement de montrer que ces signes appartiennent bien à l'autisme, mais aussi de les regrouper en «fonctions» déficientes comme la perception, l'association, l'émotion, l'imitation, la communication verbale ou non verbale...
Parallèlement, des tests neuropsychologiques récents permettent une analyse approfondie de ces troubles de la communication dans lesquels interviennent des difficultés de l'attention conjointe, de l'association différée, de l'intention, de la reconnaissance des expressions, de la conscience d'autrui... Une interprétation originale des troubles autistes est proposée par Baron-Cohen [21] et Frith [22]. Pour ces auteurs, les enfants autistes auraient difficilement ou avec retard une «théorie de la pensée », cette aptitude qui permet d'attribuer une pensée à autrui. Pour Baron-Cohen, cette aptitude qui apparaît assez tardivement dans le développement de l'enfant serait basée sur une capacité plus précoce d'anticipation et de prévision du comportement d'autrui. Cette capacité se manifesterait dès l'âge de 9 mois pour des conduites intentionnelles orientées vers l'autre, luimême considéré comme doué d'intention [22]. J.L. Adrien montre que ces troubles sont liés, au moins en partie, à une difficulté fondamentale éprouvée par l'enfant à adapter son comportement à la situation présente : le démarrage est trop brutal ou trop lent, le maintien trop labile ou trop soutenu, l'achèvement instantané ou sans fin. On dit parfois que la «modulation», la "régulation ", la «synchronisation» des comportements est perturbée [23]. Un apport important à la compréhension de l'autisme est fourni par les films ou enregistrements vidéoscopiques réalisés par les familles dès les premières semaines, donc bien avant
Tableau I

\section{ÉCHELLE D’ÉVALUATION DES COMPORTEMENTS AUTISTIQUES (ECA)}

1 - Recherche I'isolement

2 - Ignore les autres

3 - Interaction sociale insuffisante

4 - Regard inadéquat

6 - Difficultés à communiquer par les gestes et la minique

8 - Manque d'initiative, activité spontanée réduite

12 - Activités sensori-motrices stéréotypées

23 - Bizarreries de I'audition

24 - Attention difficile à fixer, détournée

L'échelle d'évaluation des comportements autistiques (ECA) comporte 29 éléments cotés de 0 à 4. Une analyse en composante principale effectuée chez 90 enfants autistes a permis d'isoler un "facteur" d'autisme [20]. Ce facteur regroupe non seulement des signes habituellement décrits dans les classifications nosographiques (1-isolement, 3-interaction sociale insuffisante) mais des éléments correspondant à 24-troubles de l'attention, 23- de la perception et 12-de la motricité. 
que le diagnostic d'autisme ait pu être évoqué. Ces études ont contribué à la mise en évidence de signes précoces dont certains peuvent être décelés dès les premiers mois. C'est un bébé trop «calme» ou parfois trop «nerveux» qui ne prête pas attention aux personnes, ne sourit pas ou sourit peu, ne paraît pas sensible à la voix, qui paraît indifférent ou "opposant" au cours de la tétée. Les troubles du sommeil sont fréquents : insomnie silencieuse ou agitée (cris jour et nuit), parfois hypersomnie [24].

L'examen systématique de ces comportements inhabituels guidé par l'échelle ECA-N retrouve les déficiences de certaines «fonctions» (figure 1). L'enfant ne manifeste pas de joie et paraît ne pas comprendre l'émotion des autres; sa communication avec eux par la voix, la mimique et les gestes est défaillante; il ne semble pas différencier les personnes. Il adopte des attitudes posturales inhabituelles et a déjà tendance à effectuer des mouvements répétés. En revanche, ses «intentions» vis-àvis des objets et des personnes ne sont pas clairement exprimées: il ne tend pas les bras à la personne qui le sollicite, il n'imite pas ses gestes, ni ses mimiques, il ne répond pas à son gazouillement. Il ne cherche pas le contact par le regard. Il paraît indifférent au monde sonore ou présente déjà des réactions paradoxales ou

\section{RÉFÉRENCES}

13. Lyonnet S, Rey F, Caillaud C, Abadie V, Munnich A, Rey J. Bases moléculaires de la phénylcétonurie en France: de l'invasion celte à la bataille de Poitiers. médecinel sciences 1988 ; 4: 544-52.

14. Chen C, Hsiao K. A chinese classic phenylketonuria manifested as autism. Br J Psychiatr 1989; 155 : 251-3.

15. Gendrot C, Ronce N, Toutain A, et al. Xlinked mental retardation exhibiting linkage to DXS 255 and PGKP1: a new MRX family (MRX 14) with localization in the pericentromeric region. Clin Genet 1994; $45: 145-53$.

16. Rousseau F, Jeitz D, Biancalana V, et al. Direct diagnosis by DNA analysis of the fragile X syndrome of mental retardation. $N$ Engl J Med 1991 ; 325 : 1673-81.

17. Warren S, Nelson D. Advances in molecular analysis of fragile $\mathrm{X}$ syndrome. $\mathrm{J} \mathrm{Am}$ Med Ass 1994; 271 : 536-42.

18. Mandel JL, Dreyfus JC. Et de sept: les répétitions nucléotidiques frappent encore! médecine/sciences $1994 ; 10$ : 472-4.

19. Curtis ARJ, Headland S, Linday S, et al. $\mathrm{X}$ chromosome linkage studies in familial Rett syndrome. Hum Genet 1993; 90 : 551-5.

20. Barthelemy C, Adrien JL, Tanguay P, et $a l$. The behavioral summarized evaluation: validity and rehability of a scale for the assessment of autistic behaviors. I Autism Dev Disord 1990; 20 : 189-204.

21. Baron-Cohen S. The autistic child's theory of mind: a case of specific developmental delay. J Child Psychol Psychiatr 1989; $30: 285-98$

22. Frith U. Autism. Explaining the enigma.

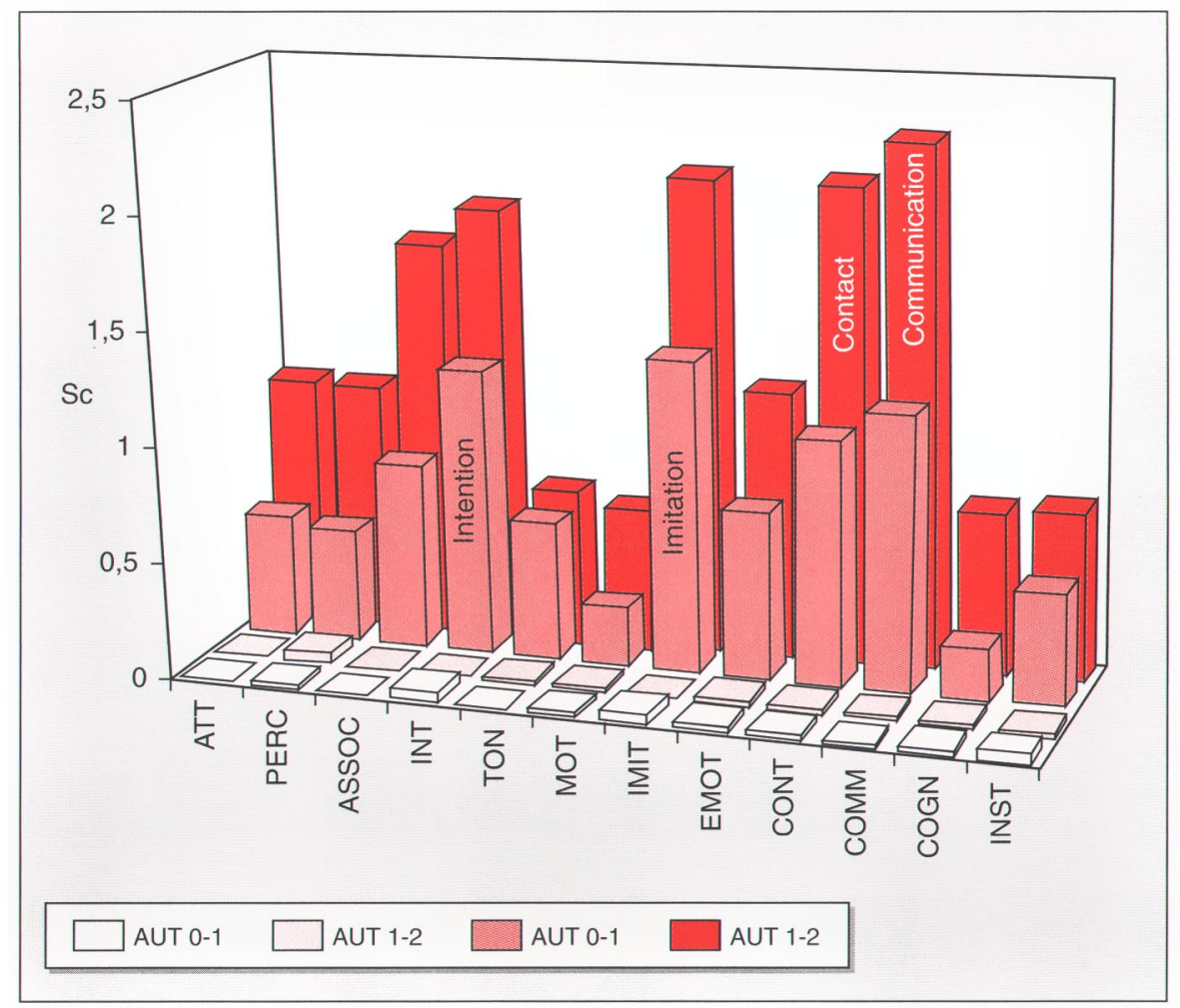

Figure 1. Le graphique montre les déficiences fonctionnelles évaluées très tôt, dès la première et la deuxième années, sur des films familiaux à l'aide de l'échelle d'évaluation des comportements autistiques du nourrisson ECA$\boldsymbol{N}$ [24]. Ces films sont enregistrés par les familles bien avant que le diagnostic d'autisme ait pu être évoqué. En abscisses les fonctions déficientes: attention (ATT), perception (PERC), association (ASSOC), intention (INT), tonus (TON), motricité (MOT), imitation (IMIT), émotion (EMOT), contact (CONT), communication (COMM), cognition (COGN), instinct (INST) (alimentation, sommeil). En ordonnées les scores à l'échelle ECA-N au cours de la première année : colonnes roses, au cours de la deuxième année: colonnes bistres. Les deux colonnes antérieures écrasées ou absentes indiquent les résultats obtenus en aveugle sur des films d'enfants témoins aux mêmes âges. On voit que les déficiences portent non seulement sur le contact et la communication, mais sur l'association, l'intention, l'imitation... 
sélectives aux sons. Enfin, souvent sont déjà présents les cris, l'irritabilité, les colères car cet enfant ne sait pas se faire comprendre. Ces différentes données suggèrent que l'autisme de l'enfant est une déficience relationnelle due à un trouble du fonctionnement du système nerveux [4]. Ce trouble affecte notamment des «systèmes de la communication" qui précèdent, dans l'ontogenèse, les «centres» du langage. Ces systèmes requièrent l'intégrité de différentes "fonctions» qui permettent à l'enfant de s'adapter aux stimulations de l'environnement.

L'ensemble de ces observations cliniques et étiologiques suggèrent que de nombreux syndromes autistes peuvent être dus à un trouble du développement du système nerveux.

\section{Explorations actuelles}

Les explorations cérébrales confirment et précisent les déficits affectant telle ou telle fonction. Nous ne rappellerons pas ici la nécessité d'un électroencéphalogramme systématique pour dépister une possible épilepsie, ni le recours fréquent à l'électrophysiologie pour confirmer un éventuel déficit auditif [5]. Nous aborderons des méthodes d'examen facultatives qui permettent cependant de préciser certains déficits fonctionnels.

\section{-Explorations neuropsychologiques}

Perception: chez l'enfant témoin, les variations de l'activité électrique cérébrale provoquées par des stimulations auditives sont, dans l'ensemble, proportionnelles à l'intensité de la stimulation. Il n'en est pas de même dans l'autisme de l'enfant, où l'on observe que des stimulations faibles peuvent provoquer des réponses de grande amplitude [25]. Ainsi est mis en évidence un trouble de la «filtration" des stimulations qui peut expliquer pourquoi l'enfant autiste, bien que donnant l'impression d'être sourd peut être perturbé par le froissement d'une feuille de papier ou par le chant d'un oiseau au dehors.

Association: chez l'enfant témoin, le couplage d'un son que l'on fait suivre peu de temps après par une lumière fait apparaître un changement dans les variations de l'activité électrique cérébrale provoquées par le son. Initialement ces variations prédominent bien évidemment sur la zone auditive. Après la répétition de quelques couples de stimulations son-lumière, une réponse au son apparaît sur l'aire visuelle [26]. C'est la manifestation d'une association croisée. Une telle association existe chez les enfants autistes mais elle est très variable. Cela permet de mieux comprendre pourquoi ces enfants lorsqu'ils regardent, semblent ne pas entendre et lorsqu'ils écoutent, semblent ne pas voir.

Langage: la mesure des débits sanguins régionaux par la méthode du SPECT (single photon emission computerized tomography) montre une réactivité particulière aux stimulations auditives dans l'autisme. Deux évaluations sont effectuées l'une au repos, l'autre pendant la répétition d'une stimula- tion auditive simple. Trois régions d'intérêt sont sélectionnées : frontale, temporale et occipitale. Un groupe d'enfants autistes est comparé à un groupe témoin d'enfants retardés. Les examens sont effectués à la demande des parents et avec l'approbation du comité d'éthique. Au repos, aucune différence n'est observée entre les deux groupes. En revanche, la stimulation auditive fait apparaître chez les enfants du groupe témoin une augmentation significative du débit sanguin cérébral dans la région temporo-occipitale gauche. Ce phénomène n'est pas observé dans le groupe des enfants autistes qui présentent, au contraire, une augmentation du débit dans la région temporo-occipitale droite (figure 2). L'hyporéactivité gauche est à rapprocher des difficultés de langage, constante chez ces enfants et l'hyperréactivité droite de leurs capacités particulières, notamment dans

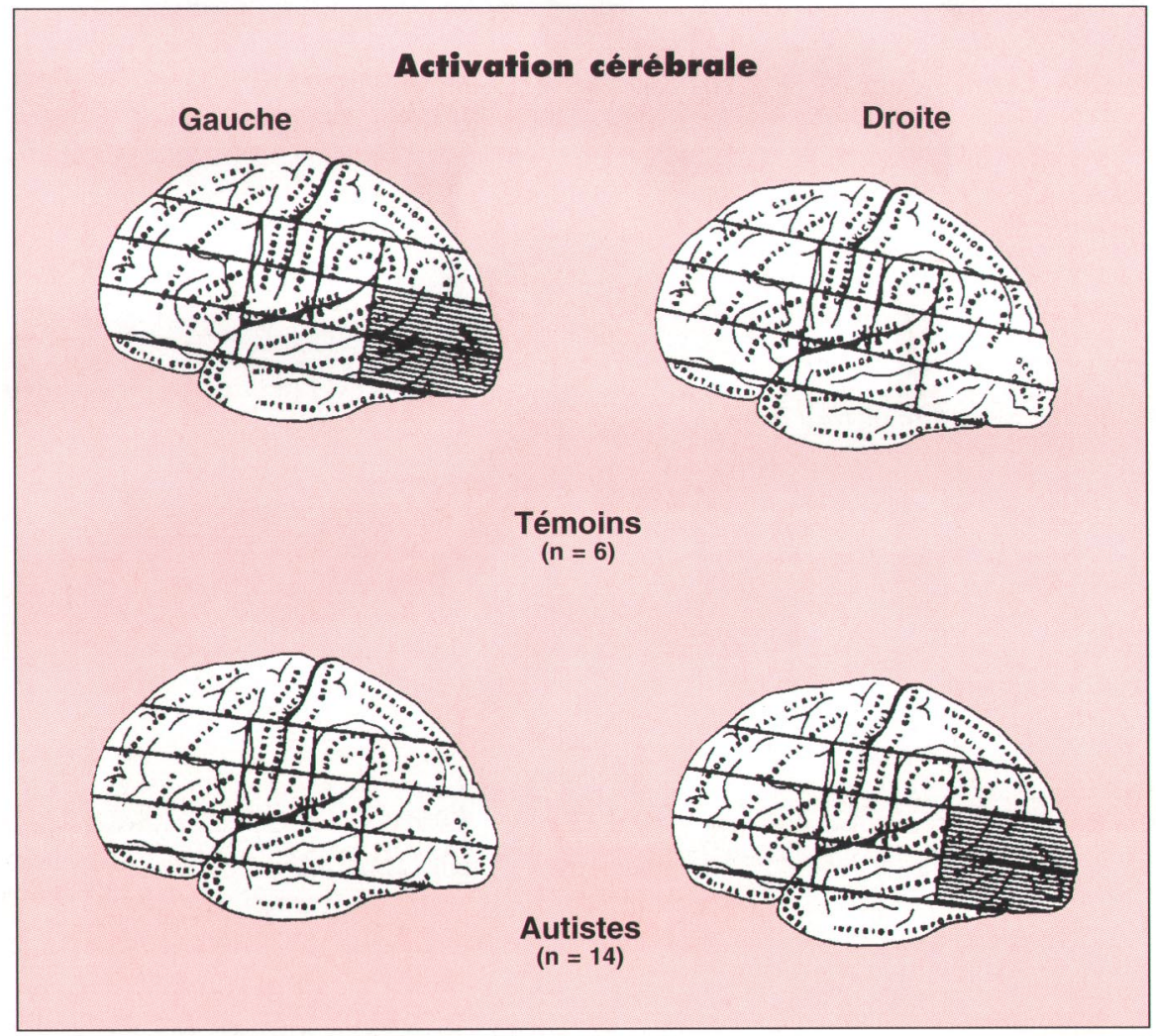

Figure 2. Modifications du débit sanguin cérébral provoquées par des stimulations auditives simples [27]. Le débit est mesuré par la méthode du SPECT (tomographie à émission de photons). Chez les enfants témoins, la stimulation auditive accroît le débit sanguin cérébral dans l'hémisphère gauche tout spécialement dans la zone du langage. Chez les enfants autistes, la même stimulation ne modifie pas le débit de l'hémisphère gauche, mais celui de I'hémisphère droit. 


\section{RÉFÉRENCES}

23. Adrien J L, Tanguay P, Barthelemy C, et al. Autistic children and the object permanence task. Acta Paedopsychiatr 1993; 56 : 2539 .

24. Sauvage D. Autisme du nourrisson et $d u$ jeune enfant, $2^{\mathrm{e}} \mathrm{ed}$. Paris: Masson, 1988.

25. Bruneau N, Garreau B, Roux S, Lelord G. Modulation of auditory evoked potentials with increasing stimulus intensity in autistic children. In: Johnson R, Parasuraman R, Rohrbaugh JW, eds. Current trends in event related potential research. EEG Clin Neurophysiol 1987; (suppl 40) : 584-9.

26. Martineau J, Roux S, Garreau B, Adrien JL, Lelord G. Unimodal and crossmodal reactivity in autism: presence of auditory evoked responses and effect of the repetition of auditory stimuli. Biol Psychiatr 1992; 31: 1190-203

27. Garreau B, Zilbovicius M, Guerin P, Samson Y, Syrota A, Lelord G. Effects of an auditory stimulation on regional cerebral blood flow in autistic children. Dev Brain Dysfunction 1994; 7 : 119-28.

28. Courchesne E, Townsend J, Saitoh O. The brain in infantile autism : posterior fossa structures are abnormal. Neurology 1994; 44: 233-41.

29. Gillberg C, Terenius C, Hagberg B, Wih $\mathrm{H}$, et al. CSF beta-endorphins in childhood neuropsychiatric disorders. Brain Dev 1990; 12: 88-92.

30. Leboyer M, Bouvard M, Recasens C, et al. Difference between plasma $\mathrm{N}$ and $\mathrm{C}$. Terminally directed B-endorphin immunoreactivity in infantile autism. Am J Psychiatr 1994; 151: 1797-801.

31. Martineau J, Barthelemy C, Hérault J, Jouve J, Muh JP. Monoamines in autistic children: a study of age-related changes. Brain Dysfunction 1991 ; 4: 141-6.

32. Herault J, Perrot A, Barthelemy C, et al. Possible association of $\mathrm{C}$ Harvey-Ras 1 (HRAS-1) marker with autism. Psychiatry Res $1993 ; 46: 261-7$.

33. Kahn A. La transmission du signal en amont et en aval de Ras. médecine/sciences 1992; 8: 1097-9.

34. Barthélémy C, Hameury L, Lelord G. Autisme de l'enfant. La thérapeutique d'échange et de développement. Paris: Expansion Scienti- le domaine visuo-spatial. De telles investigations ne peuvent faire l'objet d'examens systématiques car elles nécessitent un plateau technique important et justifient la présence auprès de l'enfant de sa famille et du médecin qui s'occupe habituellement de lui. L'application de la méthode du Doppler est plus aisée mais, pour le moment, moins précise. Elle confirme l'absence de modification du débit sanguin au niveau de l'artère sylvienne gauche chez les enfants autistes [27].

Mentionnons enfin l'existence d'anomalies morphologiques observées par Courchesne sur la partie médiane du cervelet (vermis) [28]. De telles anomalies, récemment retrouvées par notre équipe, en collaboration avec le service hospitalier Frédéric-Joliot (A. Syrota) du CEA d'Orsay, nous rappellent que le cervelet ne contrôle pas seulement la motricité, mais aussi les influx des organes des sens.

\section{- Explorations biochimiques}

Les examens biochimiques mettent en évidence des anomalies de cer- tains neurotransmetteurs comme les endorphines ou les monoamines [11]. Des anomalies du taux de $\beta$ endorphines ont été décelées chez des patients autistes dans le plasma et le LCR [29]. De plus, Leboyer et al. [30] montrent une immunoréactivité plus élevée de l'extrémité carboxyterminale de la $\beta$-endorphine dans le plasma dans un groupe d'enfants autistes comparé à un groupe témoin. Une légère diminution d'immunoréactivité est signalée aussi pour l'extrémité amino-terminale. Cette différence pourrait suggérer une anomalie du gène de la proopiomélanocortine dans l'autisme infantile. Par ailleurs, 30 à $50 \%$ des autistes présentent des troubles d'excrétion des monoamines avec anomalies des concentrations sanguines et urinaires de dopamine et sérotonine. Les études longitudinales [31] réalisées entre 3 et 12 ans ont montré que les taux d'acide homovanilique chez les enfants atteints d'autisme sont toujours supérieurs à ceux des témoins appariés (figure 3). Ces résultats biochimiques, associés à l'étude comportementale, électro-

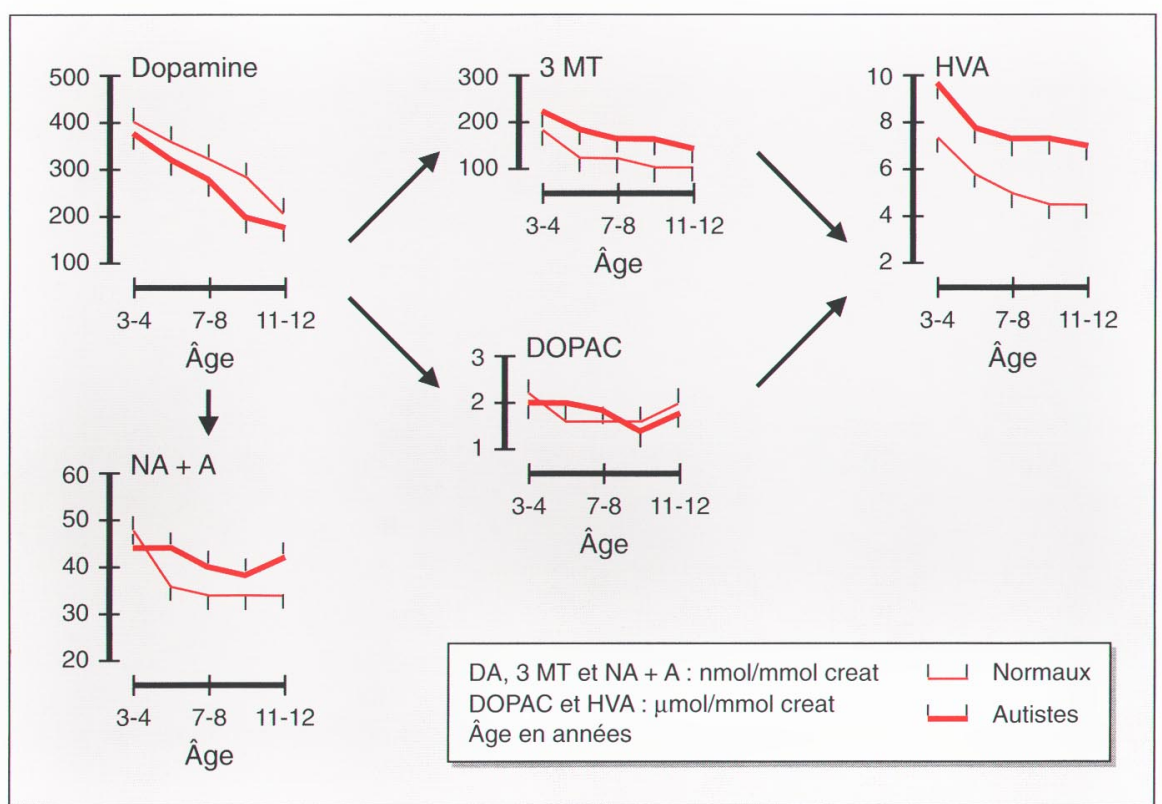

Figure 3. Dopamine et ses dérivés. 145 enfants autistes et 107 enfants témoins sont comparés [31]. En abscisses: tranches d'âges. En ordonnées: taux urinaires rapportés à la créatinine. Les taux de la dopamine sont plus faibles chez les enfants autistes, les taux de ses dérivés $=N A+A$ (noradrénaline + adrénaline), 3 MT (3 métoxytyramine), HVA (acide homovanilique) sont plus élevés chez les enfants autistes que chez les enfants témoins. II n'y a pas de différence pour le DOPAC (acide déhydroxyphénylacétique), dérivé plus labile. 
physiologique et aux données de l'imagerie, ont conduit à penser que les systèmes producteurs et de régulation de ces neuromédiateurs pouvaient être impliqués dans la physiopathologie du syndrome.

\section{- Explorations génétiques}

C'est la raison pour laquelle, en collaboration avec l'UMR 9923 CNRS de J. Mallet, nous avons entrepris des études d'association avec des gènes candidats: le gène codant pour la tyrosine hydroxylase (TH), un protooncogène voisin (H-RAS-l), les gènes de la tryptophane hydroxylase $(\mathrm{TpH})$, et de la dopamine $\beta$-hydroxylase $(\mathrm{DBH})$. Les loci correspondants sont situés en 11p15.5 sauf celui de la dopamine $\beta$-hydroxylase, localisé en 9 q34.

Dans l'étude de J. Hérault et al. [32], aucune différence de répartition allélique n'a été mise en évidence chez les autistes par rapport aux témoins pour les marqueurs des gènes des enzymes TH, TpH, DBH. En revanche, il existe une différence significative pour la répartition des allèles d'un marqueur du gène $H-R A S-1$ entre ces deux groupes (Tableau II). Quel peutêtre le sens de ce résultat? Les protéines Ras jouent un rôle essentiel dans la transduction des signaux reçus par les cellules; ces protéines régulatrices conditionnent la mise en œuvre en temps et lieu opportuns des métabolismes nécessaires à la réponse fonctionnelle cellulaire [33]. L'expression du gène RAS peut être modulée par certains facteurs de croissance nerveuse; cette expression est nécessaire pour la pérennisation de la différenciation fonctionnelle des cellules de notre organisme, notamment les cellules nerveuses. En outre, une protéine homologue d'une protéine $\mathrm{G}$, semble être impliquée dans l'apparition de la sclérose tubéreuse, maladie retrouvée associée à l'autisme. La différence allélique décrite pourrait correspondre, soit à une variété du gène H-RAS-1 considéré comme un gène de susceptibilité, soit à la présence au voisinage du locus $\mathrm{H}-R A S$ d'un gène muté, à ce jour inconnu, intervenant dans la croissance et/ou les fonctions du SNC. Ces résultats amènent à étudier d'autres gènes (voir bibliographie dans [32]) et à explorer les gènes situés en 11p15.5 qui participent à la régulation de la neurotransmission (gène codant pour un récepteur de la dopamine de type $\mathrm{D} 4$ ) et de la croissance du système nerveux.

\section{Orientations thérapeutiques}

Ces progrès peuvent-ils modifier le traitement des enfants autistes? Pour ce qui concerne la génétique moléculaire, la réponse est, au moins pour le court terme, négative. L'autisme n'est pas une seule "maladie» héréditaire selon des modes de transmission classiques. De longues recherches seront encore nécessaires pour préciser les différents facteurs, parmi lesquels les anomalies géniques, qui participent à ce trouble du développement du système nerveux.

Actuellement, les traitements médicamenteux de l'autisme sont essentiellement symptomatiques. De nombreux psychotropes ont été utilisés. Suivant les cas, les sédatifs peuvent améliorer la turbulence et l'excitation, les stimulants la passivité, l'inactivité ou au contraire l'instabilité, des remèdes agonistes ou antagonistes des endorphines l'agitation et l'agressivité. Les traitements antiépileptiques appliqués lorsque les crises sont présentes et/ou lorsqu'une impulsivité avec colères imprévisibles sont associées à un tracé EEG très perturbé peuvent, non seulement modifier le tracé, mais améliorer le comportement. Mentionnons enfin des études systématiques effectuées sur plusieurs années en doubleaveugle sur l'effet de la vitamine B6 associée au magnésium. Ces études regroupant des résultats cliniques, électrophysiologiques et biochimiques montrent que l'amélioration clinique observée chez certains enfants coïncide avec la «normalisation" des réponses EEG et des concentrations des dérivés périphériques des neurotransmetteurs (dopamine, sérotonine) [31]. En fait, les traitements de l'autisme sont aujourd'hui encore essentiellement des psychothérapies éducatives au sens le plus large. Cependant, les progrès neurophysiologiques dans la connaissance des déficits observés ont permis d'élaborer des «thérapies d'échange et de développement», qui ont pour objet l'amélioration des

\begin{tabular}{|lcccc|}
\hline \multicolumn{5}{c|}{ Tableau II } \\
\hline Gènes & Allèles & Normaux & Autistes & Chi 2 \\
\hline$T p H$ & A1 & 0,60 & 0,62 & \\
& A2 & 0,40 & 0,38 & 0,02 \\
DBH & A1 & 0,64 & 0,74 & \\
& A2 & 0,36 & 0,26 & 1,89 \\
& B1 & 0,36 & 0,33 & 0,09 \\
TH & B2 & 0,64 & 0,67 & \\
& B & 0,26 & 0,28 & 0,03 \\
$H-R A S$ & b & 0,74 & 0,72 & 9,64 \\
& B1 & 0,14 & 0,18 & \\
INS & B2 & 0,10 & 0,25 & 0,008 \\
& B3 & 0,76 & 0,57 & 0,14 \\
IFG2 & A1 & 0,84 & 0,81 & \\
& A2 & 0,16 & 0,19 & 0,51 \\
\hline
\end{tabular}

Fréquence allélique de différents gènes intervenant dans la neurotransmission et/ou le développement du système nerveux [32]. 72 enfants autistes sont comparés à 72 enfants témoins. On ne relève pas de différence significative pour les allèles des gènes Tph (tryptophane hydroxylase), qui intervient dans le métabolisme de la sérotonine, DBH (dopamine hydroxylase), TH (tyrosine hydroxylase) qui participent respectivement à la dégradation et à la production de dopamine, INS (insuline) et IGF2 (insulin-like growth factor-2). En revanche, une différence statistiquement significative est observée pour les allèles du gène RAS. Notons que les gènes H-RAS, TH, INS, IGF2 sont tous situés sur le bras court du chromosome 11, dans la région 11p15.5. 
dysfonctionnements évalués par la clinique et même par l'électrophysiologie [34]. Ces rééducations fonctionnelles sont réalisées dans une ambiance psychothérapique à la fois chaleureuse et détendue, en liaison, et même en collaboration étroite, avec la famille. Elles reposent sur trois principes: (1) la tranquillité, qui permet à l'enfant de se repérer dans le temps et dans l'espace en favorisant la filtration des messages de l'environnement; (2) la disponibilité, qui favorise les associations et les acquisitions non plus seulement avec le corps propre (mouvements monotones répétés inlassablement) mais avec le monde des objets et des personnes; (3) la réciprocité, notamment dans le jeu du ballon, de la poupée et les jeux d'imitation de gestes, de mimiques, voire de paroles et de chants avec le thérapeute, qui facilite la communication. Ces thérapies sont précédées par une période d'observation d'un mois au moins qui a pour but l'évaluation neurophysiologique et neuropsychologique des «fonctions» déficitaires (attention, perception, association, posturomotricité, imitation...).

La séance de thérapie individuelle dure généralement 20 minutes. Elle est effectuée chaque jour de part et d'autre d'une table dans un local aménagé à cet effet, mais aussi dans le bureau de l'orthophoniste, du psychologue, dans le local de psychomotricité, de kinésithérapie ou dans la pataugeoire. De plus, les principes généraux de la thérapie peuvent se retrouver en situation de groupe ou dans la famille. Cette thérapie est poursuivie pendant au moins une année scolaire; elle peut être renouvelée deux ou trois ans si cela est nécessaire. Elle est accompagnée et suivie par des évaluations régulières. Soixante-quinze enfants ont été traités selon cette méthode depuis 10 ans. Les évaluations, qu'elles soient individuelles ou qu'elles concernent des groupes (valeurs moyennes portant sur les cotations des échelles) font état de progrès très appréciables. Une comparaison systématique a porté sur deux groupes d'enfants: le premier bénéficie pendant l'année scolaire de l'ensemble des soins spécialisés délivrés dans le service, le second des mêmes soins associés aux thérapies individuelles d'échange et de développement. Les valeurs moyennes des cotations montrent une amélioration plus rapide et plus importante dans le second groupe. Au total, ces thérapies permettent de «débrouiller» l'enfant, de transformer les relations avec son entourage avant d'aborder des thérapies et rééducations plus spécifiques (orthophoniques, psychomotrices, pédagogiques et scolaires, psychanalytiques ou de la méthode TEACCH).

A ce propos, il n'est pas possible de passer sous silence le fait que ces enfants peuvent progresser tout au long de leur vie, notamment sur le plan de la socialisation s'ils bénéficient des conditions favorables et de constater que ces conditions ne sont pas remplies actuellement ni pour les adolescents ni pour les adultes.

\section{Conclusion}

En conclusion, comme cela est habituel en médecine, du fait des progrès des hypothèses, méthodes et techniques d'exploration, la conception même de l'autisme se trouve profondément modifiée. Les méthodes nouvelles d'exploration ont mis en évidence des anomalies portant sur les «comportements » électrique, circulatoire et métabolique cérébraux, et qui témoignent d'une perturbation globale du fonctionnement du système nerveux. Ces données peuvent contribuer à la connaissance des déficits de la communication. La précocité d'apparition des troubles, l'existence possible d'antécédents pathologiques de la grossesse, voire de particularités géniques retrouvées chez certains enfants, plaident en faveur d'un trouble du développement.

Cela n'amène pas à méconnaître l'importance de facteurs psychologiques. On sait que le pronostic des infirmités motrices est lié pour une bonne part à la motivation de l'enfant comme à l'aide constante et éclairée de sa famille. Cette aide, que les parents apportent sans compter, s'avère particulièrement utile pour les médecins dans les insuffisances relationnelles de l'autisme. Mais il apparaît désormais que ces insuffisances relationnelles doivent, comme les infirmités motrices, bénéficier aussi des progrès récents issus de l'étude du développement du système nerveux.

\section{TIRÉS À PART}

G. Lelord.

\section{Summary}

Neurobiology of childhood autistic syndromes

Childhood autism is characterized by social withdrawal, impairment in communication and bizarre responses to the environment. It has been for a long time considered that this syndrome and its difficulties in relating to others can be imputed to conscious or inconscious errors of the mother. Epidemiological and neurobiological researches showing association of autistic syndromes with well known genetic and/or metabolic diseases, as well as clinical works exhibiting very early symptoms support the hypothesis of a developmental disorder of the nervous system. Cartography and imagery cerebral examinations demonstrated difficulties in the modulation of perceptive responses, in the stabilisation of cross-modal associative responses and in the reactivity of the left hemisphere to auditive stimulations. Abnormalities in monoamines and endorphines metabolism were also observed. Recent studies comparing groups of autistic with groups of normal children showed some peculiarities in a gene (Harvey-Ras) involved in the regulation of neurotransmission and cells development. These preliminary results have no therapeutical consequences. On the opposite, physiological data on cerebral functioning can guide the "exchange and development therapies ", educational psychotherapies which contribute to the improvement of these children. 